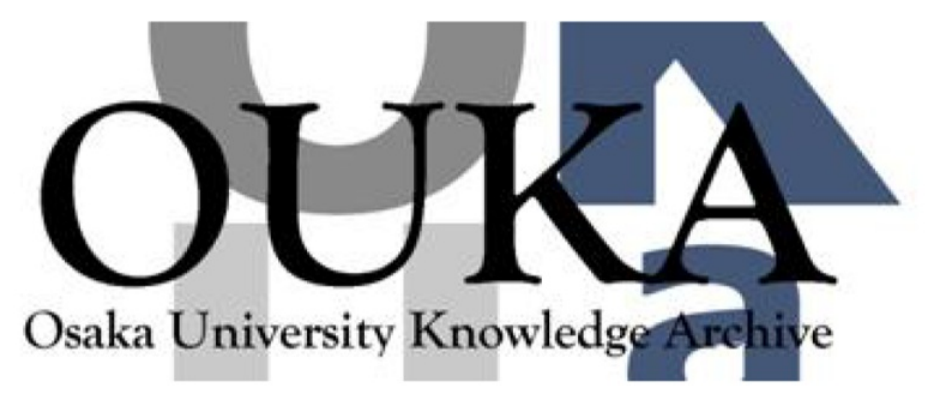

\begin{tabular}{|c|l|}
\hline Title & $\begin{array}{l}\text { Optical tuning and switching of photonic defect } \\
\text { modes in cholestericliquid crystals }\end{array}$ \\
\hline Author(s) & $\begin{array}{l}\text { Yoshida, Hiroyuki; Lee, Chee Heng; Miura, } \\
\text { Yusuke et al. }\end{array}$ \\
\hline Citation & $\begin{array}{l}\text { Applied Physics Letters. 90(7) p. 071107- } \\
\text { p.071107 }\end{array}$ \\
\hline Issue Date & $2007-02-15$ \\
\hline oaire:version & VoR \\
\hline URL & https://hdl.handle.net/11094/75660 \\
\hline rights & \\
\hline Note & \\
\hline
\end{tabular}

Osaka University Knowledge Archive : OUKA

https://ir. Library. osaka-u. ac. jp/

Osaka University 


\section{Optical tuning and switching of photonic defect modes in cholestericliquid crystals}

Cite as: Appl. Phys. Lett. 90, 071107 (2007); https://doi.org/10.1063/1.2560805

Submitted: 11 December 2006 . Accepted: 16 January 2007 . Published Online: 15 February 2007

Hiroyuki Yoshida, Chee Heng Lee, Yusuke Miura, Akihiko Fujii, and Masanori Ozaki

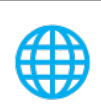

\section{ARTICLES YOU MAY BE INTERESTED IN}

Low-threshold and high efficiency lasing upon band-edge excitation in a cholesteric liquid crystal

Applied Physics Letters 90, 091114 (2007); https://doi.org/10.1063/1.2710777

Polymer stabilized liquid crystal films reflecting both right- and left-circularly polarized light Applied Physics Letters 93, 201901 (2008); https://doi.org/10.1063/1.3003869

Electrically color-tunable defect mode lasing in one-dimensional photonic-band-gap system containing liquid crystal

Applied Physics Letters 82, 3593 (2003); https://doi.org/10.1063/1.1577829

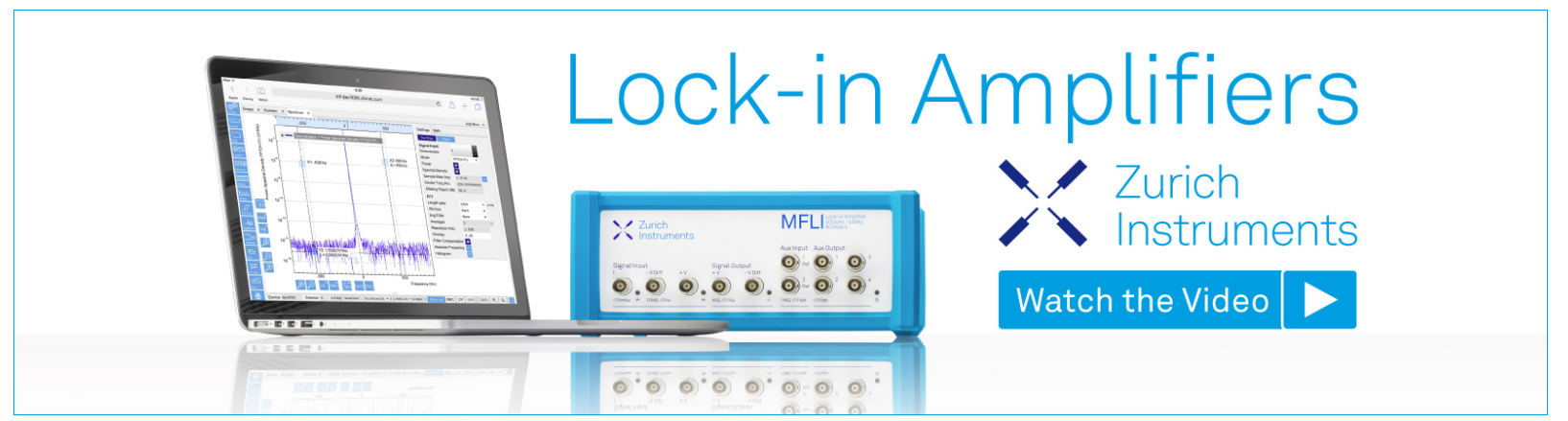




\title{
Optical tuning and switching of photonic defect modes in cholesteric liquid crystals
}

\author{
Hiroyuki Yoshida, ${ }^{\text {a) }}$ Chee Heng Lee, Yusuke Miura, Akihiko Fujii, and Masanori Ozaki ${ }^{\text {b) }}$ \\ Department of Electrical, Electronic and Informational Engineering, Graduate School of \\ Engineering, Osaka University, 2-1 Yamada-oka, Suita, Osaka 565-0871, Japan
}

(Received 11 December 2006; accepted 16 January 2007; published online 15 February 2007)

\begin{abstract}
Optical tuning of photonic defect modes in cholesteric liquid crystals is demonstrated based on a contrast in the pitch length. Two-photon laser lithography was performed in a cholesteric helix to leave an unpolymerized region in the center to act as a structural defect. The unpolymerized region was then substituted by an azobenzene dye-doped cholesteric liquid crystal which exhibits a pitch shortening upon ultraviolet exposure. Reversible shifting or on-off switching of the defect mode was realized as a result of selectively inducing a pitch shortening of the cholesteric liquid crystal at the defect and changing the contrast in the pitch length between the bulk and defect. (C) 2007 American Institute of Physics. [DOI: 10.1063/1.2560805]
\end{abstract}

Cholesteric liquid crystals (ChLCs) have recently attracted interest as self-organizing, tunable photonic-band-gap (PBG) materials. ${ }^{1,2}$ The chiral constituents doped in a conventional nematic LC host material cause the molecules to self-organize into a helical periodic structure. Due to the chiral periodicity of the LC director, they exhibit a $100 \%$ selective reflection for circularly polarized light with the same circular handedness as the material, in the wavelength region described by $\lambda=n_{o} p \sim n_{e} p$, where $n_{o}$ and $n_{e}$ are the ordinary and extraordinary refractive indices, and $p$ is the periodicity of the ChLC called the pitch. ${ }^{3}$ Utilizing the PBG property where the group velocity reaches zero at the photonic-bandedge wavelengths, laser action has been demonstrated in ChLCs. ${ }^{4}$

The controllability of the photonic properties has been one of the advantages of fabricating a photonic device from ChLCs. This is realized by utilizing the external field sensitivity of the LC molecules or certain dopants to induce a change in the refractive indices or the helical pitch. Tuning of the PBG and lasing wavelength has been demonstrated upon thermally controlling the ChLC pitch ${ }^{5}$ or by fabricating a spatial modulation of the ChLC pitch. ${ }^{6,7}$ On the other hand, stabilization of the helix was demonstrated in ChLC standing films with doped photoreactive monomers which polymerize upon ultraviolet (UV) light irradiation. ${ }^{8}$ One particularly interesting and useful tuning method is by means of an external optical field. Photoinduced trans-cis isomerization in the azobenzene dye doped in ChLCs was found to cause either an elongation or shortening of the ChLC pitch. Reversible modulation of the PBG was demonstrated in ChLCs doped with various azobenzene derivatives, upon UV exposure. ${ }^{9-12}$

A defect mode with high wavelength selectivity is realized in the usually nontransmitting PBG by introducing a structural defect in the perfect helical lattice. While there are many possibilities as to the medium to act as the defect such as an isotropic dielectric layer ${ }^{13}$ or a phase shift in the LC director, ${ }^{14,15}$ an easily tunable defect mode is realized by introducing a different ChLC material in the defect. In such a configuration, the defect-mode wavelength is determined by

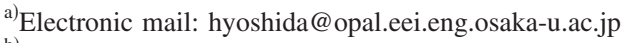

${ }^{b)}$ Electronic mail: ozoaki@opal.eei.eng.osaka-u.ac.jp
}

the contrast of the pitch lengths at the defect and bulk, ${ }^{16,17}$ and an optically tunable defect mode is realized if the ChLC at the defect can selectively be pitch modulated by an external optical field. In this letter, such optical tuning of the photonic defect mode will be demonstrated. The proposed structure was fabricated by a laser-induced two-photon polymerization method described in Ref. 18 so that an unpolymerized ChLC was left between two polymerized ChLC layers, and then substituting the unpolymerized $\mathrm{ChLC}$ by an azobenzene dye-doped ChLC (azo-ChLC) to act as the photoresponsive defect medium (Fig. 1). Because the polymerized ChLC does not show photochromism, only the azo$\mathrm{ChLC}$ at the defect is selectively pitch modulated by UV exposure.

The sample used in experiment was a right-handed, photopolymerizable ChLC mixture with Irgacure 819 as the photoinitiator (prepared by Merck KGaA). $0.6 \mathrm{wt} \%$ of 4-(dicyanomethylene)-2-methyl-6-(4-dimethylaminostyryl)4H-pyran (Excition: DCM) dye was added in the mixture for observation purposes during device fabrication. The ChLC material was infiltrated in a LC cell with cell gap of $\sim 6 \mu \mathrm{m}$ at $75^{\circ} \mathrm{C}$ and cooled slowly to room temperature, after which laser lithography was performed. The laser intensity and scan speed used for the lithography process were $0.79 \mathrm{GW} \mathrm{cm}^{-2}$ and $126 \mu \mathrm{s} \mu \mathrm{m}^{-2}$, respectively, yielding an approximate polymer thickness of $2.7 \mu \mathrm{m}$ [observed on a scanning elec-
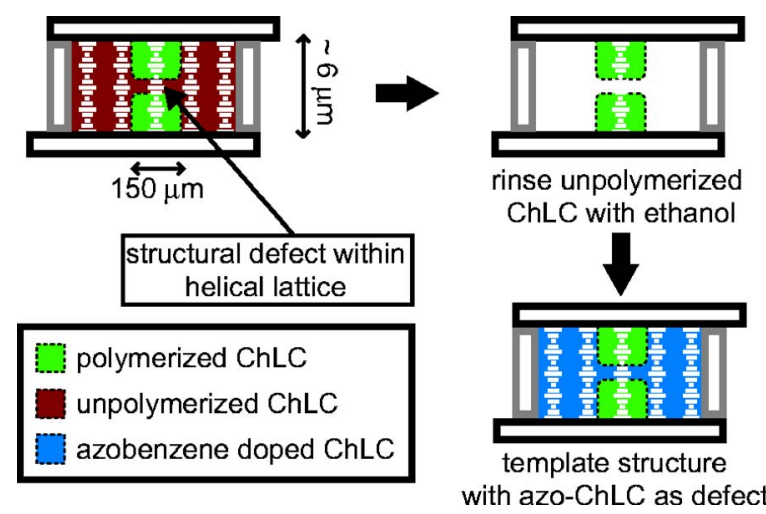

FIG. 1. (Color online) Experimental procedure to introduce a photoreactive structural defect in the ChLC material for UV optical switching. 


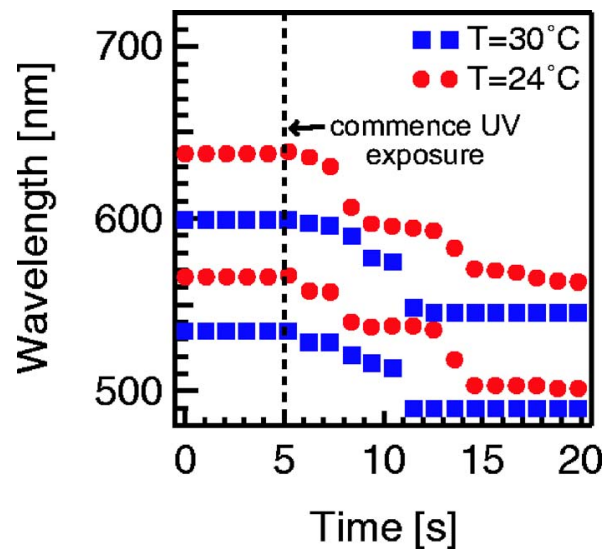

FIG. 2. (Color online) Spectral position of the PBG of the azo-ChLC upon UV exposure at two different temperatures.

tron microscope (Hitachi: S-2100)]. The azo-ChLC to act as the defect medium was composed of a nematic LC host (Merck: E-44), 30 wt \% right-handed chiral dopant (Merck: R-811), and 3.8 wt \% azo dye (Tokyo Chemical Industry: 4-diethylaminoazobenzene) and was infiltrated in the structure after rinsing the unpolymerized ChLC by ethanol. The transmittance spectrum of the device was measured on a microscope (Nikon: Optiphot 2, numerical aperture of 0.5, measurement spot $\phi \sim 20 \mu \mathrm{m})$ upon right-circularly polarized light incidence by a charge coupled device multichannel spectrometer (Hamamatsu Photonics: PMA-11). The change in the transmittance was recorded as an UV $(\lambda<437 \mathrm{~nm})$ light from a high-pressure mercury lamp was irradiated coaxially on the sample at $\sim 17 \mathrm{~mW} \mathrm{~cm}^{-2}$.

Figure 2 shows the spectral position of the PBG of the azo-ChLC in a conventional planar cell upon UV exposure, for two different temperatures at $T=24$ and $30{ }^{\circ} \mathrm{C}$. The azo$\mathrm{ChLC}$ exhibited a shorter pitch at a higher temperature, and the UV exposure also caused a pitch shortening within several seconds of commencing. The large shortening of the pitch in a relatively small time span is attributed to the fast photochemical reaction of the azobenzene dye. The pitch shortening was also discrete due to the strong anchoring at the substrate surfaces: upon UV exposure, a domain core with a discretely shortened pitch started to grow and the transmittance changed once the domain grew over the spot at which the transmittance was measured.

Figure 3 shows the transmittance spectrum (at $30^{\circ} \mathrm{C}$ ) of the fabricated structure with the azo-ChLC in the defect. Within the PBG of the polymerized ChLC (elongated by laser lithography ${ }^{17,18}$ ) exhibited at $\lambda_{c} \sim 611 \mathrm{~nm}$, a single defect mode was observed at $\lambda \sim 632 \mathrm{~nm}$ due to the pitch and refractive indices contrast at the defect. Upon UV exposure, the defect mode blueshifted and settled at $\lambda \sim 620 \mathrm{~nm}$ within $6 \mathrm{~s}$ of irradiation. The time-resolved study of the defectmode wavelength showed that the shift commenced almost simultaneously with UV exposure, supporting that the tuning was caused by the photochromic pitch shortening of the azoChLC. The results also showed that only a small shortening of the pitch occurred (a single discrete shortening of pitch corresponding to a single discrete defect-mode shift exhibited at time $\sim 9.4 \mathrm{~s}$ ) compared to a conventional cell, under the same exposure conditions. This is attributed to the less amount of UV reaching the azo-ChLC at the defect because of the absorption of the DCM dye in the polymer ChLC

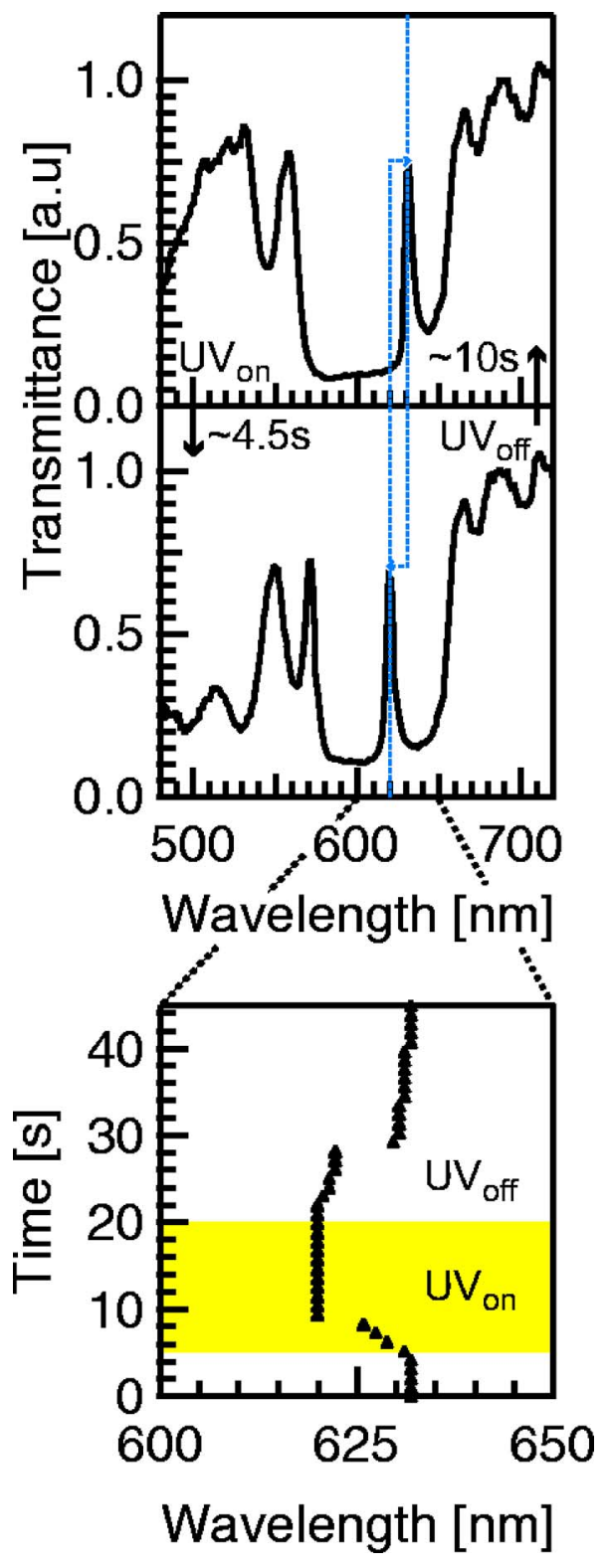

FIG. 3. (Color online) Transmittance spectrum of the fabricated structure at $30^{\circ} \mathrm{C}$ upon UV exposure and the time-resolved measurement of the defect-mode wavelength.

layers. The polymer ChLC surfaces may also have affected the pitch-shortening behavior of the azo-ChLC due to the weaker anchoring: a weaker anchoring enables a gradual shortening of the azo-ChLC pitch (as such observed within $3 \mathrm{~s}$ of UV exposure) until a certain point is reached where it becomes energetically preferable for the pitch to shorten discretely and match the surroundings (within the LC cell of size $\sim 1.8 \times 1.8 \mathrm{~cm}^{2}$, the structure is only $\sim 150 \times 150 \mu \mathrm{m}^{2}$ ). The defect mode returned to its original wavelength after $\sim 10$ s of UV cutoff due to a relaxation process to the thermally preferred trans state. The optical tuning had high reproducibility, with the transmittance showing no decline even after repetitive UV irradiation.

Figure 4 shows the transmittance spectrum of the fabricated structure at $24{ }^{\circ} \mathrm{C}$, in which no defect modes are initially exhibited because of the spectral overlap between the PBG of the azo-ChLC and that of the polymerized ChLC (numerical calculations support that this should be the case even when the refractive indices of the two ChLCs 

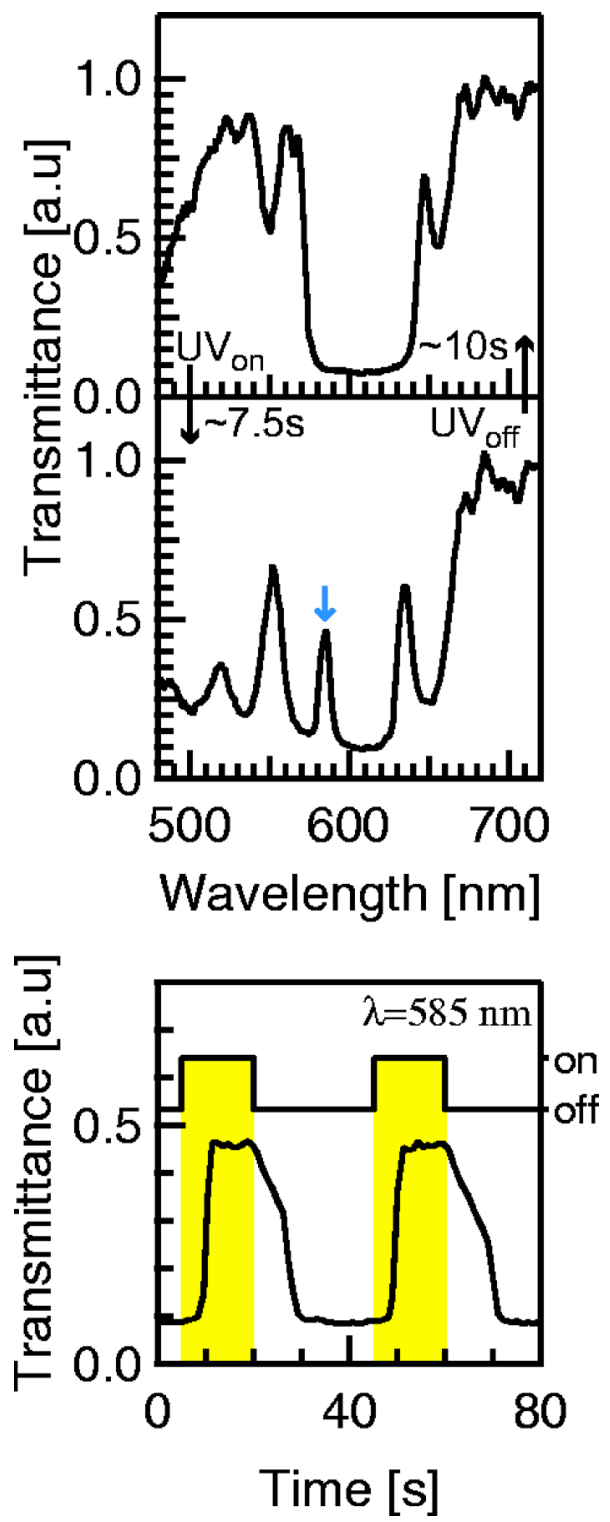

FIG. 4. (Color online) Transmittance spectrum of the fabricated structure at $24{ }^{\circ} \mathrm{C}$ upon UV exposure and the time-resolved measurement of the transmittance at the defect mode.

do not match). Upon UV exposure, a defect mode appeared at $\lambda \sim 587 \mathrm{~nm}$ and settled at $585 \mathrm{~nm}$ after the pitch-shortened domain grew over the measurement spot and a pitch contrast was induced between the bulk and the defect; at cutoff the defect mode first exhibited a small redshift and then disappeared at $\lambda \sim 590 \mathrm{~nm}$. Therefore, an on-off switching of the defect mode is performed. The fact that the switching is not completely discrete may also be a result of the weaker anchoring of the ChLC polymers and is reflected in the timeresolved measurement of the transmittance at $\lambda \sim 585 \mathrm{~nm}$, where a fast and a slow component corresponding to the gradual redshift and the discrete disappearing of the defect mode are observed in the off switching. This mode of optical switching was also reversible and had high reproducibility.

Both wavelength tuning and on-off switching of the defect mode have been demonstrated in an electrode-free configuration by optically inducing a contrast of the ChLC pitch between the bulk and defect. Further optimization of the structure such as the defect layer width and the materials should enable realization of a faster photonic device with a high $Q$, which may, combined with its simple and controlled fabrication process, be useful for optical sensor or limiter applications or as tunable narrow bandpass filters in integrated optical circuits.

The authors thank Merck KGaA for the photopolymerizable ChLC mixture. This work is partly supported by a Grant-in-Aid for Scientific Research from the Ministry of Education, Culture, Sports, Science and Technology of Japan.

${ }^{1}$ E. Yablonovitch, Phys. Rev. Lett. 58, 2059 (1987).

${ }^{2}$ S. John, Phys. Rev. Lett. 58, 2486 (1987).

${ }^{3}$ H. deVries, Acta Crystallogr. 4, 219 (1951).

${ }^{4}$ V. I. Kopp, B. Fan, H. K. M Vithana, and A. Z. Genack, Opt. Lett. 23, 1707 (1998).

${ }^{5}$ K. Funamoto, M. Ozaki, and K. Yoshino, Jpn. J. Appl. Phys., Part 2 42, L1523 (2003).

${ }^{6}$ Y. Huang, Y. Zhou, and S. T. Wu, Appl. Phys. Lett. 88, 011107 (2006).

${ }^{7}$ Y. Huang, Y. Zhou, C. Doyle, and S. T. Wu, Opt. Express 14, 1236 (2006).

${ }^{8}$ T. Matsui, R. Ozaki, K. Funamoto, M. Ozaki, and K. Yoshino, Appl. Phys. Lett. 81, 3741 (2002).

${ }^{9}$ E. Sackmann, J. Am. Chem. Soc. 93, 7088 (1971).

${ }^{10}$ S. Kurihara, K. Matsumoto, and T. Nonaka, Appl. Phys. Lett. 73, 160 (1998).

${ }^{11}$ P. V. Shibaev, R. L. Sanford, D. Chiappetta, V. Milner, A. Genack, and A. Bobrovsky, Opt. Lett. 13, 2358 (2005).

${ }^{12}$ S. Kurihara, Y. Hatae, T. Yoshioka, M. Moritsugu, T. Ogata, and T. Nonaka, Appl. Phys. Lett. 88, 103121 (2006).

${ }^{13}$ Y.-C. Yang, C.-S. Kee, J.-E. Kim, H.-Y. Park, J.-C. Lee, and Y.-J. Jeon, Phys. Rev. E 60, 6852 (1999).

${ }^{14}$ M. Ozaki, R. Ozaki, T. Matsui, and K. Yoshino, Jpn. J. Appl. Phys., Part 1 42, 472 (2003).

${ }^{15}$ J. Schmidke, W. Stille, and H. Finkelmann, Phys. Rev. Lett. 90, 083902 (2003).

${ }^{16}$ T. Matsui, M. Ozaki, and K. Yoshino, Phys. Rev. E 69, 061715 (2004).

${ }^{17}$ H. Yoshida, C.-H. Lee, A. Fujii, and M. Ozaki, Appl. Phys. Lett. 89, 231913 (2006).

${ }^{18}$ H. Yoshida, C.-H. Lee, Y. Matsuhisa, A. Fujii, and M. Ozaki, Adv. Mater. (Weinheim, Ger.) (to be published). 